\title{
INOVAÇÃO ATRAVÉS DA GESTÃO DA ROTINA: UMA ESTRATÉGIA PARA PEQUENAS E MÉDIAS EMPRESAS
}

\author{
José Edson Guimarães Júnior'; Alex Álisson Bandeira Santos² \\ 1 PARANAPANEMA; Via do Cobre 3700 (Area Industrial Oeste), Dias d'Avila, BA, \\ 42850-000; guimar.jr@gmail.com \\ 2 SENAI CIMATEC, Avenida Orlando Gomes, 1845, Piatã, Salvador-Bahia, Cep: \\ 41.650-010.
}

Resumo: A inovação se constitui como uma importante ferramenta para as empresas na busca pela diferenciação no mercado e competitividade. Os setores de P\&D têm recebido cada vez mais recursos para desenvolver projetos, e o governo tem fomentado a inovação, quer seja através de renúncia fiscal, subvenção ou financiamento de projetos junto a universidades e centros de pesquisa. Porém fica uma questão: será que a inovação é atividade presente no dia-a-dia das empresas industriais de menor porte? Muitas empresas de destaque no cenário nacional apontam a gestão da rotina como base para melhoria do desempenho e até inovação; será que há uma correlação entre inovação e gerenciamento da rotina? Neste contexto, o estudo tem por objetivo investigar a correlação entre inovação e gerenciamento da rotina.

Palavras-Chave: Gestão do conhecimento; inovação; Gestão da rotina.

\section{INNOVATION THROUGH ROUTINE MANAGEMENT: A STRATEGY FOR SMALL AND MEDIUM-SIZED ENTERPRISES}

\begin{abstract}
Innovation is an important tool for companies in the search for market differentiation and competitiveness. The P\&D sectors have received more and more resources to develop projects, the government has fomented innovation, whether through tax breaks, subsidy or financing by funding to universities and research centers. But there is one question: is innovation an activity present in the day-to-day of smaller industrial companies? Many prominent companies in the national scenario point to the management of the routine as a basis for improving performance and even innovation; Is there a correlation between innovation and routine management? In this context, the study aims to investigate the correlation between innovation and routine management.
\end{abstract}

Keywords: Knowledge management, innovation, management routine. 


\section{INTRODUÇÃO}

No atual cenário macroeconômico, onde a competitividade é a palavra de ordem, a inovação se constitui como principal ferramenta na busca de diferenciação e garantia de sobrevivência das empresas. Neste contexto, as pequenas e médias empresas (PME), que de acordo com a pesquisa PINTEC 2008 (IBGE, 2010) [1] representam $20 \%$ do PIB e $60 \%$ dos empregos formais, necessitam de um esforço adicional para competir em nível global e encontrar formas de serem mais eficientes e inovadoras.

No Brasil, as micro e pequenas empresas brasileiras (MPMEs) representam $99 \%$ do total de empresas e são responsáveis por $56 \%$ da força de trabalho formal e cerca de $24 \%$ do PIB do País [2] e é sabido que muitos recursos financeiros não são aproveitados pelas MPMEs de forma a gerar inovações para o país [3]. Os números apresentados não mudaram tanto ao longo do tempo [4]. Através de dados do IBGE [1], chegamos à conclusão que o investimento em Pesquisa e Desenvolvimento está sendo empregado, em sua grande maioria, pelas grandes empresas, e isto se deve ao fato que as micro, pequenas e médias empresas apresentarem atraso tecnológico, baixa competitividade e ênfase na produção de bens de menor valor agregado (commodities) [5]. Portanto, o advento de um sistema de gestão simples e que possibilite às PMEs a geração do conhecimento para inovação trará ganhos tanto para as empresas, quanto para o crescimento do país.

\subsection{Gerenciamento da Rotina}

O sistema de gestão da rotina nada mais é do que a aplicação de um método gerencial que auxilia no estabelecimento de metas de desempenho, comparação com outros participantes do mercado e por fim, a padronização dos processos produtivos e/ou de serviços, transformando-os em conhecimento organizacional de modo a proporcionar um monitoramento contínuo dos resultados e guiando a organização até os melhores resultados operacionais da categoria, o que, na visão de [6], trata-se, de um sistema de gestão de desempenho, conjugando informações financeiras e nãofinanceiras para a tomada de decisão e ação gerencial, visando influenciar o comportamento dos subordinados. Segundo [7], o gerenciamento da rotina tem como princípios os seguintes pontos: definição da autoridade e responsabilidade de cada um na organização; padronização dos processos; acompanhamento e controle do desempenho dos processos; ação corretiva nos processos na ocorrência de desvios de desempenho quando comparados com as metas; participação dos empregados; bom ambiente de trabalho; melhoria contínua.

A gestão da rotina proposta por [7], estabelece um ciclo lógico para aplicação da metodologia, apoiado no PDCA (plan, do, check, act) de [8] e para inicar sua aplicação, o autor postula que é necessário estabelecer metas de desempenho. $O$ 
exercício para estabelecimento de metas deve estar diretamente ligado ao planejamento estratégico da organização, sua visão e missão. As metas surgem de lacunas operacionais, e essas podem ser oriundas de um desempenho abaixo do esperado ou pode ser a criação de um novo patamar de desempenho. Uma vez estabelecidas as metas, o próximo passo será a priorização, levando-se em conta as metas que se apresentam mais aderentes à estratégia definida pela organização.

Para concluir o estabelecimento de metas, é necessário garantir o envolvimento e o compromisso de todos os níveis da organização com as metas definidas e priorizadas. Este objetivo será alcançado no momento em que todas as metas forem desdobradas desde a alta gerência até o chão de fábrica e os indicadores de performance das metas estejam associados a todos nos respectivos níveis.

A continuidade da implementação do gerenciamento da rotina, segundo [9] ocorre através da elaboração de um plano de ação para alcançar as metas estabelecidas. Todo o plano de ação pressupõe uma análise prévia e este é o ponto chave do método proposto para a gestão da rotina. Nesta etapa surge a oportunidade de entender melhor o desafio associado a meta, comparar desempenhos de organizações competidoras e/ou que atuam em mercados similares e propor novas soluções. O exercício de analisar os motivos pelos quais a meta ainda não foi alcançada gera a inovação, que não necessariamente tem que ser um invento ou a criação de algo completamente novo definido como inovação radical por [10], podendo ser a introdução de pequenas modificações e/ou alterações na aplicação de máquinas, materiais, classificada como inovação incremental, segundo a mesma autora. De acordo com [9], pode-se dividir o método de análise em 3 tipos: Análise Funcional, Análise do Fenômeno e Análise do Fluxo de processo.

Como parte final da implementação do gerenciamento da rotina, a padronização complementa a metodologia e transforma todas as informações obtidas através das análises e discussões entre os envolvidos. Segundo [11] este procedimento permite a conversão do conhecimento tácito em conhecimento explícito, o que se constituiu como a base para a gestão do conhecimento na escola japonesa. Alguns estudos tratam da gestão da rotina como também a inovação em PMEs [12] [13], porém de forma não correlacionada.

Uma vez estabelecido o plano e as metas, incluindo o desdobramento das mesmas, reuniões de acompanhamento serão conduzidas periodicamente, de modo a verificar o cumprimento das ações, atingimento de resultados e envolvimento de todos na gestão.

\section{METODOLOGIA}

Como desenvolvimento do trabalho, fez-se necessária uma avaliação do estado atual de inovação das empresas industriais da região. Devido ao grande número de empresas industriais instaladas na região metropolitana de Salvador (RMS), optou-se por avaliar o perfil das indústrias da cidade de Lauro de Freitas, uma vez que, dentre todas as cidades que formam a RMS, ela apresentou maior crescimento conforme pesquisa [14]. 
Lauro de Freitas é um município situado na região metropolitana de Salvador e que possui população estimada em 156.936 habitantes. O município está em crescimento acelerado e apresenta dados expressivos no cenário nacional, tais como o segundo PIB que mais crescia no país e era o quarto município que mais gerou empregos em 2009 [15].

Para execução da pesquisa, foi adotado o método survey, que segundo [16], refere-se ao uso de questionário estruturado dado a uma amostra de uma população e destinado a coletar informações específicas dos entrevistados. Para tanto, foi elaborado um questionário estruturado contendo questões fechadas do tipo dicotômicas, múltipla escolha e escalonadas. O conteúdo das questões foi embasado no referencial teórico ([1], [17]). Após esboçado, o questionário foi avaliado e validado numa aplicação piloto junto a uma empresa industrial de médio porte e posteriormente foi enviado via e-mail e disponibilizado na internet como um formulário eletrônico para preenchimento. O questionário baseou-se nos objetivos de avaliar a capacidade de inovação das pequenas e médias empresas industriais; entender mecanismos utilizados pelas PMEs da região para inovação e investigar a existência de correlação entre o gerenciamento da rotina e inovação.

O questionário foi aplicado entre os meses de abril e julho do ano de 2012 (dados somente disponibilizados recentemente). Ao todo foram 63 contatadas, das quais 21 enviaram respostas, perfazendo uma taxa de resposta da ordem de 33,33\%. O questionário pode ser segmentado em 3 partes: a) caracterização da empresa, b) posicionamento quanto a inovação, c) gestão do conhecimento

Para responder ao questionamento quanto a correlação entre gestão da rotina e inovação, recorreu-se a um modelo probabilístico econométrico denominado de logit. O modelo logit é um modelo econométrico e de seleção qualitativa [18], baseado numa função de probabilidade logística acumulada, a saber:

$$
P_{i}=F\left(Z_{i}\right)=F\left(b_{0}+\sum_{i=1}^{n} b_{i} \cdot X_{i}\right)=\frac{1}{1+e^{-Z_{i}}}=\frac{1}{1+e^{-\left(b_{0}+\sum_{i=1}^{n} b_{i} \cdot X_{i}\right)}}
$$

onde:

$P_{i}=$ probabilidade de ocorrência de um evento, dada à ocorrência de $\mathrm{X}$ na observação i. $1 \leq \mathrm{i} \leq \mathrm{k} \quad \mathrm{k}=$ número de observações; ${ }^{b}{ }=$ coeficiente da variável independente $X^{i} ; Z^{i}=$ índice contínuo teórico determinado pelas variáveis explicativas $X^{i}$, sendo:

$$
\begin{aligned}
& Z_{i}=b_{0}+\sum_{i=1}^{n} b_{i} \cdot X_{i}, \text { que conduz à seguinte expressão: } \\
& \frac{P_{i}}{1-P_{i}}=\frac{1+e^{b_{0}+\sum_{i=1}^{n} b_{i} \cdot X_{i}}}{1+e^{-\left(b_{0}+\sum_{i=1}^{n} b_{i} \cdot X_{i}\right.}}=e^{b_{0}+\sum_{i=1}^{n} b_{i} \cdot X_{i}}
\end{aligned}
$$


Logo, conclui-se:

A variável dependente é o logaritmo relacionando a probabilidade de ocorrência de um dos dois possíveis eventos. A principal característica do modelo logit é transformar o problema de predição probabilística em um intervalo [0,1].

Uma vez justificada a escolha do município, foi realizada uma pesquisa no banco de dados de empresas da cidade e selecionadas as empresas industriais. Devido à dificuldade em obter respostas das empresas contatadas, optou-se por utilizar $15 \%$ de erro da amostra e risco de $15 \%$, resultando numa amostra com 21 empresas, sendo 17 pequenas empresas e 4 médias empresas. A definição das empresas entrevistas deu-se através de escolha aleatória, realizada com auxílio do software Microsoft Excel.

\section{RESULTADOS E DISCUSSÃO}

Com base na metodologia, respostas advindas dos questionários e auxílio dos softwares Minitab, versão15.1.1.0 e Excel, versão 2007, obtiveram-se os seguintes resultados:

Caracterização da amostra: A amostra foi composta de empresas pertencentes aos setores de atuação: montagem (33,3\%); metalurgia (25\%); alimentícia (10\%); têxtil (10\%); gráfica (10\%); mineração (5\%); moveleira (5\%) e de plástico $(5 \%)$.

No que se refere ao porte das empresas que compuseram a amostra e considerando o critério de classificação do porte de acordo com a quantidade de empregados, utilizado pelo Sebrae (2012), obteve-se a seguinte distribuição: pequenas empresas $(83,3 \%)$ e médias empresas $(16,7 \%)$. Em relação ao tempo de operação, as empresas foram distribuídas de acordo com o exposto na tabela 1 .

Tabela 1- Distribuição das empresas pelo tempo de operação

\begin{tabular}{l|l} 
Faixas & $\begin{array}{l}\text { Tempo de } \\
\text { operação }\end{array}$ \\
\hline menor que 5 & 3 \\
\hline entre 5 e 10 & 4 \\
\hline entre 10 e 15 & 5 \\
\hline entre 15 e 20 & 2 \\
\hline entre 20 e 25 & 2 \\
\hline maior que 25 & 5 \\
\hline
\end{tabular}

FONTE: Elaborado pelos autores

No quesito inovação, obtiveram-se os seguintes dados: $83,3 \%$ das empresas respondentes afirmaram que inovaram nos últimos 12 meses, enquanto somente $16,7 \%$ não implementaram qualquer inovação no referido período. Foram analisados 
os tipos de inovação implantadas, tendo como resultado os seguintes números: $76 \%$ dos entrevistados declararam ter implantado inovações no processo produtivo, 43\% em sistemas de gestão, $52 \%$ com novos produtos, $52 \%$ inovaram em melhorias de produto, $10 \%$ implantaram inovações em marketing e 14\% declararam que não inovaram, conforme pode ser visualizado na figura 1.

Aprofundando mais os dados sobre inovação, das empresas entrevistadas, somente $14 \%$ declararam ter depositado patente para produtos.

No que se refere a aplicação da gestão da rotina e baseado no referencial teórico já abordado, convencionou-se que as empresas que praticassem: resolução de problemas (8D, FMEA, PDCA, dentre outras ferramentas), auditoria do cumprimento da rotina e padronização e ou acompanhamento da produção diários, seriam classificadas como utilizadoras da metodologia de gestão da rotina, enquanto as demais seriam classificadas como não praticantes do gerenciamento da rotina.

Figura 1- Tipos de inovação

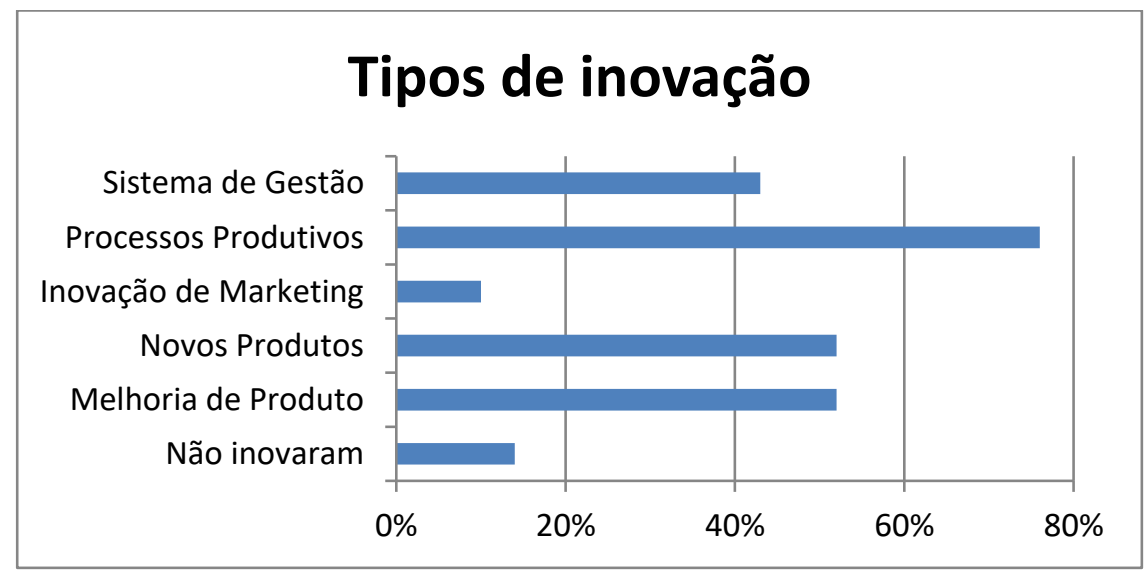

FONTE: Elaborado pelos autores

Deste modo obteve-se uma distribuição onde 67\% aplicam e 33\% não aplicam ferramentas do gerenciamento da rotina e na figura 2 é possível observar a o tipo de ferramenta utilizada pelas empresas industriais pesquisadas.

Para a apresentação de dados, utilizou-se o logit para pesquisar a correlação entre variáveis obtidas das respostas dos questionários com a inovação. As variáveis de trabalho foram listadas na tabela 2.

Como os dados de trabalho eram atributivos, optou-se por fazer o estudo de regressão logística utilizando o modelo econométrico logit, tendo o software Minitab R15 como base para os cálculos estatísticos.

Processou-se o modelo e identificando que a variável que a maior correlação com a variável resposta era GR, já que o valor P-value $(0,083)$, vide Tabela 3 , era menor que os demais. Logo, evidenciou-se uma correlação positiva entre a aplicação de ferramentas de gestão da rotina (GR) e a inovação (Inov), já que o coeficiente é positivo (5.77). 
Figura 2 - Ferramentas de gerenciamento da rotina

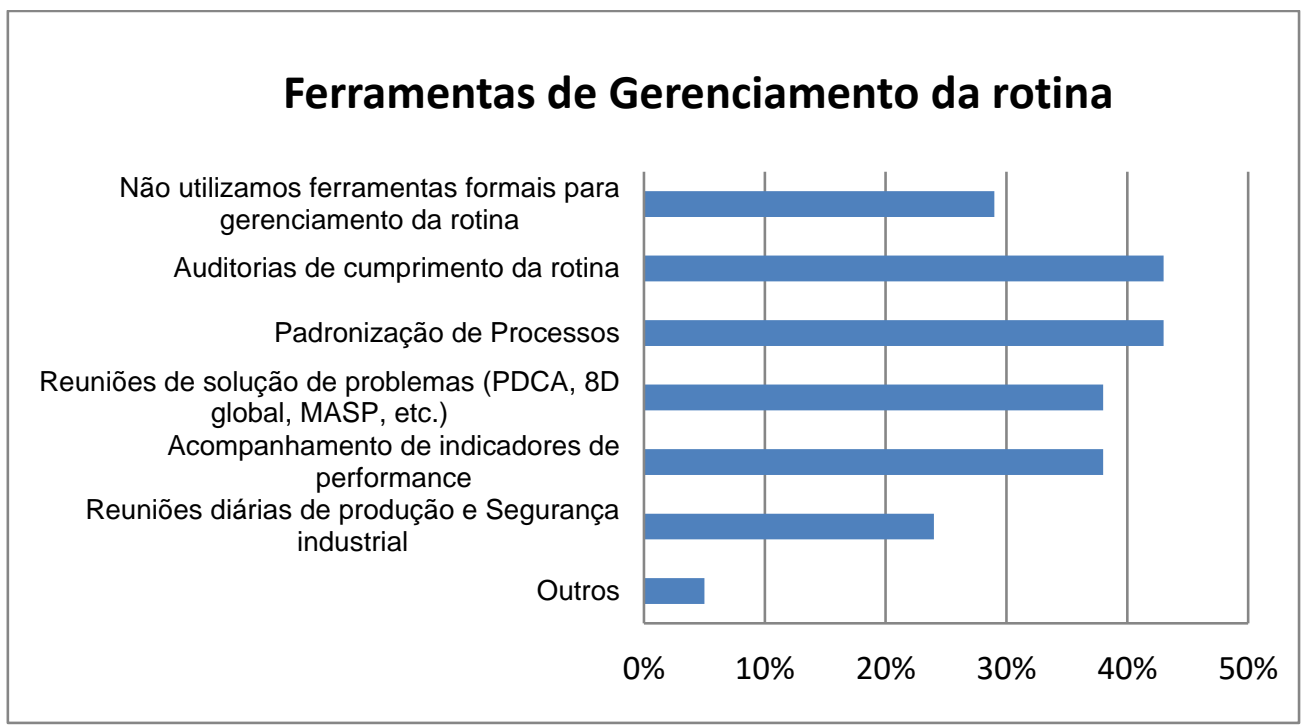

FONTE: Elaborado pelos autores

Tabela 2 - Tabela de variáveis utilizados no modelo Logit

\begin{tabular}{|c|c|c|c|c|}
\hline $\begin{array}{l}\text { Tipo de } \\
\text { variável }\end{array}$ & Variável & Código & Medidas & Tipo \\
\hline \multirow{2}{*}{$\begin{array}{c}\text { Variável } \\
\text { dependente }\end{array}$} & \multirow{2}{*}{ Inovação } & \multirow{2}{*}{ INOV } & $\begin{array}{l}\text { A empresa inovou nos últimos } \\
12 \text { meses }=1\end{array}$ & \multirow{2}{*}{ Atributo } \\
\hline & & & $\begin{array}{l}\text { A empresa não inovou nos } \\
\text { últimos } 12 \text { meses }=0\end{array}$ & \\
\hline \multirow{5}{*}{$\begin{array}{l}\text { Variáveis } \\
\text { explicativas }\end{array}$} & \multirow{2}{*}{$\begin{array}{l}\text { Uso da } \\
\text { Gestão da } \\
\text { rotina }\end{array}$} & \multirow[t]{2}{*}{ GR } & $\begin{array}{l}\text { A empresa usa ao menos uma } \\
\text { ferramenta de gerenciamento da } \\
\text { rotina }=1\end{array}$ & \multirow[t]{2}{*}{ Atributo } \\
\hline & & & $\begin{array}{l}\text { A empresa não usa ferramentas } \\
\text { de gerenciamento da rotina }=0\end{array}$ & \\
\hline & \multirow{2}{*}{$\begin{array}{l}\text { Certificação } \\
\text { em normas de } \\
\text { qualidade }\end{array}$} & \multirow{2}{*}{ Sist_gest } & $\begin{array}{l}\text { A empresa é certifica em pelo } \\
\text { menos uma norma de qualidade } \\
=1\end{array}$ & \multirow[t]{2}{*}{ Atributo } \\
\hline & & & $\begin{array}{l}\text { A empresa não é certificada em } \\
\text { normas da qualidade }=0\end{array}$ & \\
\hline & $\begin{array}{l}\text { Tempo de } \\
\text { operação da } \\
\text { empresa }\end{array}$ & Tempo & Tempo de operação empresa: & Contínua \\
\hline
\end{tabular}

FONTE: Elaborado pelos autores

O p-value do teste foi de 0,05 e todas as outras variáveis apresentaram p-value dos respectivos coeficientes acima de 0,05. De onde pode-se concluir que, para estes 
dados analisados, a inovação é explicada, com 95\% de confiança, pela aplicação de ferramentas da gestão da rotina.

Tabela 3 - Logit para Inovação x Gestão da inovação

\begin{tabular}{c|c|c|c|c|c|c|c}
$\begin{array}{c}\text { Varíaveis } \\
\text { explicativas }\end{array}$ & Coef. & SE coef & $\mathbf{Z}$ & $\begin{array}{c}\text { P- } \\
\text { value }\end{array}$ & $\begin{array}{c}\text { Odds } \\
\text { Ratio }\end{array}$ & $\begin{array}{c}\text { Intervalo de } \\
\text { confiança = 95\% } \\
\text { Lower } \\
\text { Upper }\end{array}$ \\
\hline Constant & $-2,7368$ & 2,24262 & $-1,22$ & 0,222 & - & - & - \\
\hline GR & 5,77261 & 3,32686 & 1,74 & 0,083 & 321,38 & 0,47 & 218220,24 \\
\hline tempo & 0,28144 & 0,188838 & 1,49 & 0,135 & 1,33 & 0,92 & 1,92 \\
\hline sist_gest & $-4,8749$ & 3,39726 & $-1,43$ & 0,151 & 0,01 & 0 & 5,95 \\
\hline
\end{tabular}

FONTE: Elaborado pelos autores

\section{CONSIDERAÇÕES FINAIS}

Aparentemente excludentes, inovação e gerenciamento da rotina estão ligados e existem fortes evidências para afirmar que a aplicação dos conceitos da qualidade total conduz as organizações à inovação. As empresas japonesas foram e ainda são sinônimos de inovação e qualidade, contrariando o dilema custo $x$ qualidade $e$ revolucionando a gestão da manufatura. Consoante a esta afirmativa, o estudo estatístico, através do modelo econométrico logit, evidenciou que a inovação nas empresas industriais do município de Lauro de Freitas pode ser explicada pelo fator aplicação de ferramentas de gerenciamento da rotina tais como: gestão à vista, método de análise e solução de problemas, acompanhamento frequente de produção e não tem correlação significativa com fatores como: implantação de certificações ISO, tempo de operação, número de empregados.

Esta conclusão é respaldada por [19] que afirma que aplicação de ferramentas de qualidade conduz a organização à inovação, porém deve-se levar em consideração a existência de ambientes onde há uma maior necessidade de aplicação de métodos e ferramentas de gerenciamento da rotina com mais rigor do que outras, citando a manufatura como um exemplo destes ambientes. Diferentemente da conclusão obtida por [20], não foi observada uma correlação entre o tempo de operação da empresa e a sua inovação.

Uma vez justificada a relação entre gerenciamento da rotina e inovação, podese concluir que a aplicação de ferramentas simples e que demandam uma forte interação entre os membros das empresas se constituem como uma estratégia viável para o aumento de competitividade das pequenas e médias empresas (PMEs).

\section{REFERÊNCIAS}

1

Pesquisa Industrial de Inovação Tecnológica 2010. Rio de Janeiro: IBGE. Disponível em: <http://www.ibge.gov.br >. Acesso em: 10 dez. 2012. 
2INÁCIO JÚNIOR, E. Padrões de inovação em pequenas e médias empresas e suas implicações para o desempenho inovativo e organizacional. 2008. $272 \mathrm{f}$. Tese (Doutorado em política científica e tecnológica) - Instituto de Geociências, Universidade Estadual de Campinas, São Paulo, 2008.

${ }^{3}$ LA ROVERE, R. Perspectivas das micro, pequenas e médias empresas no Brasil. Revista de Economia Contemporânea, v.5, p.137-154, 2001. Número especial.

${ }^{4}$ SERVIÇO BRASILEIRO DE APOIO À MICRO E PEQUENAS EMPRESAS. Pequenos Negócios em Números. Disponível em: $<$ http://www.sebrae.com.br/Sebrae/Portal\%20Sebrae/UFs/SP/Pesquisas/dados mpe

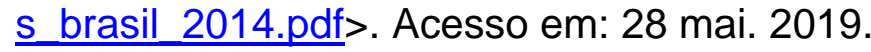

${ }^{5}$ DE NEGRI, J.; SALERNO, M.; CASTRO, A. Inovações, padrões tecnológicos e desempenho das firmas industriais brasileiras. In: DE NEGRI, J.; SALERNO, M. (Org.). Inovações, padrões tecnológicos e desempenho das firmas industriais brasileiras. Brasília: Ipea, 2005. 713p.

${ }^{6}$ WAAL, A. Behavioral factors important for the successful implementation and use of performance measurement systems. Management Decision, v. 41, n. 8, p. 688-697, 2003.

${ }^{7}$ CAMPOS, V. F. Gerenciamento da rotina do trabalho do dia-a-dia. Nova Lima: INDG Tecnologia e Serviços Ltda., 2004.

${ }^{8}$ SHEWHART, W. Statistical method from the viewpoint of quality control. New York: Dover, 1986.

${ }^{9}$ FALCONI, V. O Verdadeiro Poder. Nova Lima: INDG Tecnologia e Serviços Ltda., 2009.

${ }^{10}$ ESTREIN, J. Estreitando a lacuna da inovação. São Paulo: DVS Editora, 2010.

${ }^{11}$ NONAKA, I.; TAKEUCHI, H. Criação de conhecimento na empresa: como as empresas japonesas geram a dinâmica de inovação. Rio de Janeiro: Campus,1997.

${ }^{12 M O R A E S, ~ M . B . ~ d e ; ~ C A M P O S, ~ T . M . ; ~ L I M A, ~ E . ~ M o d e l o s ~ d e ~ d e s e n v o l v i m e n t o ~ d a ~}$ inovação em pequenas e médias empresas do setor aeronáutico no Brasil e no Canadá. Gestão \& Produção, v. 26, n. 1, e2002, 2019.

${ }^{13}$ SILVA, S.S.S.; LUCENA, E.A. Como os gestores têm aprendido sobre a rotina de gerenciamento do processo produtivo dos pedidos dos clientes? Gestão \& Produção, v. 22, n. 2, p.356-369, 2015.

${ }^{14}$ SUPERINTENDÊNCIA DE ESTUIDOS ECONÔMICOS E SOCIAIS DA BAHIA. PIB municipal. Salvador: SEl. Disponível em: 
$<$ http://www.sei.ba.gov.br/index.php?option=com_content\&view=article\&id=128\&ltem id=88>. Acesso em: 07 abr. 2012

${ }^{15} \mathrm{CHINESES} \mathrm{interessados} \mathrm{em} \mathrm{grande} \mathrm{projeto} \mathrm{hoteleiro.} \mathrm{Tribuna} \mathrm{da} \mathrm{Bahia,} \mathrm{Salvador,}$ 27 set. 2010.Disponível em: <http://www.tribunadabahia.com.br/2010/09/27/chinesesinteressados-em-grande-projeto-hoteleiro >. Acesso em: 07 abr. 2012.

${ }^{16}$ MALHOTRA, N. K. Pesquisa de marketing: uma orientação aplicada. 3. ed. Porto Alegre: Bookman, 2001.

${ }^{17}$ TIRONI, L. F.; CRUZ, B. O. Inovação incremental ou radical: há motivos para diferenciar? Uma abordagem com dados da PINTEC. Texto para Discussão. Rio de Janeiro: Instituto de Pesquisa Econômica Aplicada. n. 1360, out. 2008.

${ }^{18}$ PINDYCK, R. S.; RUBINFELD, D. L. Econometric models and economic forecasts. Boston: Irwin McGraw-Hill, 1998.

${ }^{19}$ ASHKENAS, R. It's time to rethink continuos improvement. Harvard Business Review Blog Network. 8 maio 2012. Disponível em: $<$ http://blogs.hbr.org/ashkenas/2012/05/its-time-to-rethink-continuous.html>. Acesso em: 15 jun. 2012.

20NATÁRIO, M; COUTO, J; SOUSA, M. Grau de Inovação das Pequenas e Médias Empresas nas Regiões Periféricas da Guarda e Açores. Centro de Estudos de Economia Aplicada do Atlântico: Working Paper, n. 10. dez, 2010. Disponível em: $<$ http://www.ceeapla.uac.pt/uploads/pms/attachments/Paper10-2010.pdf>. Acesso em: 13 jun. 2012. 